\title{
Andersen-Tawil syndrome (LQT7) is associated with alterations in left ventricular rotational mechanics
}

\author{
Anita Kalapos*, Róbert Sepp, Péter Domsik, Tamás Forster, Attila Nemes \\ Second Department of Medicine and Cardiology Centre, Medical Faculty, University of Szeged, \\ Hungary
}

The goal: Andersen-Tawil syndrome (ATS), also called Andersen Syndrome and Long QT Syndrome 7 (LQT7) is a rare condition. ATS is characterized by episodic flaccid muscle weakness, distinctive dysmorphic features, ventricular arrhythmias and prolonged QT interval in the presence of normal conventional left ventricular (LV) structural and functional parameters. In the normal heart, the LV base rotates clockwise, while the apex rotates counterclockwise during systole, producing a "towel-wringing" motion of the heart. The net difference between the LV base and LV apex is called "net twist angle". The present study was designed to assess LV rotational mechanics in ATS by three-dimensional speckle-tracking echocardiography (3DSTE).

Patients and Methods: The study comprised 6 patients with ATS, their results were compared to 20 healthy volun-

\section{Received: $18^{\text {th }}$ Apr 2014}

*Address for correspondence: Second Department of Medicine and Cardiology Centre, University of Szeged, Korányi fasor 6, H-6720 Szeged, Hungary Phone: +36-62-545220

E-mail: nemes.attila@med.u-szeged.hu teers. Complete two-dimensional Doppler echocardiography and 3DSTE were performed in all cases. 3DSTE was used to measure apical and basal LV rotations and LV twist.

Results: During 3DSTE, apical LV rotation proved to be $8.43 \pm 1.54$ degree, while basal LV rotation was $-2.31 \pm 1.31$ degree, therefore LV twist was $11.12 \pm 5.32$ degree in healthy subjects. In 3 out of 6 ATS patients, LV basal and LV apical rotation were in the same direction resulting in the near absence of LV twist showing „rigid body rotation”. In the remaining 3 ATS patients, LV apical rotation $(2.06 \pm 1.30$ degree, $p<0.05)$ together with LV twist $(6.51 \pm 1.28$ degree, $p<0.05)$ were significantly decreased as compared to controls. Mean basal rotation showed no significant difference compared to the values of controls $(-4.45 \pm 1.87$ degree, $p$ $=\mathrm{NS}$ ).

Conclusions: Significant alterations in LV rotational mechanics could be demonstrated in ATS by 3DSTE.

KEYWORDS: three-dimensional, speckle-tracking, echocardiography, left ventricular mechanics, AndersenTawil syndrome.

CITATION: Cardiol Croat. 2014;9(5-6):188.

\section{Literature}

1. Nemes A, Kalapos A, Domsik P, Forster T. Three-dimensional speckle-tracking echocardiography -- a further step in non-invasive three-dimensional cardiac imaging. Orv Hetil. 2012;153:1570-7. 